Schultheiß, Birgit; Breuel, Lars; Maiwald, J.; Matthäi, H.; Ning, Gangmin; Obstoj, Peter; Henning, Günter:

Beurteilung der hämodynamischen Wirksamkeit von Extrasystolen mittels Impedanzkardiographie und peripherer Pulswellenanalyse

Zuerst erschienen in: Biomedizinische Technik = Biomedical Engineering. Berlin [u.a.] : de Gruyter. - 45 (2000), S1, S. 253-254.

Erstveröffentlichung: $\quad 2000$

Datum Digitalisierung: 2009-07-17

ISSN (online): $\quad$ 1862-278X

ISSN (print): $\quad$ 0013-5585

DOI: $\quad 10.1515 /$ bmte.2000.45.s1.253

[Zuletzt gesehen: 2019-08-20]

„Im Rahmen der hochschulweiten Open-Access-Strategie für die Zweitveröffentlichung identifiziert durch die Universitätsbibliothek IImenau. "

"Within the academic Open Access Strategy identified for deposition by IImenau University Library."

„Dieser Beitrag ist mit Zustimmung des Rechteinhabers aufgrund einer (DFG-geförderten) Allianz- bzw. Nationallizenz frei zugänglich."

"This publication is with permission of the rights owner freely accessible due to an Alliance licence and a national licence (funded by the DFG, German Research Foundation) respectively."

\section{DFG}

Nationallizenzen 


\title{
BEURTEILUNG DER HÄMODYNAMISCHEN WIRKSAMKEIT VON EXTRASYSTOLEN MITTELS IMPEDANZKARDIOGRAPHIE UND PERIPHERER PULSWELLENANALYSE
}

\author{
B. Schulthei ${ }^{1}$, L. Breuel ${ }^{2}$, J. Maiwald ${ }^{3}$, H. Matthäi ${ }^{3}$, G. Ning ${ }^{1}$, P. Obstoj ${ }^{1}$, G. Henning ${ }^{1}$ \\ ${ }^{1}$ Institut für Biomedizinische Technik und Medizinische Informatik, TU Ilmenau, Deutschland \\ ${ }^{2}$ medis $\mathrm{GmbH}$, Ilmenau, Deutschland \\ ${ }^{3}$ Klinik für Innere Medizin IV, Friedrich-Schiller-Universität Jena, Deutschland
}

E-mail: bsc@informatik.tu-ilmenau.de

\section{EINLEITUNG}

In der klinischen Routine ist gegenwärtig das Langzeit-EKG zur Beurteilung der klinischen Relevanz von Arrhythmien und Extrasystolen Mittel der Wahl. Obwohl bekannt ist, daß ventrikuläre (VES) und auch supraventrikuläre (SVES) Extrasystolen sowohl ein normales, ein reduziertes und teilweise überhaupt kein Schlagvolumen besitzen können, wird gegenwärtig die hämodynamische Wirksamkeit von arrhythmogenen Schlägen in der klinischen Routine wenig beachtet. Nachfolgend wird eine Methode vorgestellt, die eine quantitative Einschätzung der hämodynamischen Wirksamkeit von SVES und VES gestattet.

\section{MATERIALIEN UND METHODEN}

Bei 63 Patienten mit klinisch relevanten Arrhythmien wurden EKG, kardiale und periphere pulssynchrone Impedanzänderung mit dem Meßsystem multiscreen (medis $\mathrm{GmbH}$ ) kontinuierlich über $3-5 \mathrm{~h}$ aufgezeichnet. Für die am Thorax, am Unterarm sowie Unterschenkel erfaßten Impedanzänderungen wurden Kurvenformanalysen durchgeführt und verschiedene Amplituden- und Zeitparameter automatisch bestimmt.

Anhand von Amplituden- und Formparametern aus dem EKG erfolgte eine online-Vorklassifikation von VES, welche im Anschluß an die Datenerfassung nach automatischer Clusterung interaktiv validiert werden kann. Zur SVES-Klassifikation wurde ein neuartiger histogrammbasierter Algorithmus eingesetzt.

\section{ERGEBNISSE}

Bei den klinischen Untersuchungen wurde eine sehr große interindividuelle Variabilität bezüglich der hämodynamischen Wirksamkeit von arrhythmogenen Schlägen festgestellt. So konnten sowohl SVES mit stark reduziertem Schlagvolumen (Abb. 1) als auch VES mit sehr guter hämodynamischer Wirksamkeit (Abb. 2) sowie ventrikuläre Extrasystolen mit reduziertem bzw. ohne erkennbares Schlagvolumen (Abb. 3) beobachtet werden. Aufgrund der kompensatorischen Pause und der daraus resultierenden besseren Füllung des linken Vorhofes weist der direkt auf die Extrasystole folgende Normalschlag oft ein erhöhtes Schlagvolumen auf (Abb. 1 und 3).

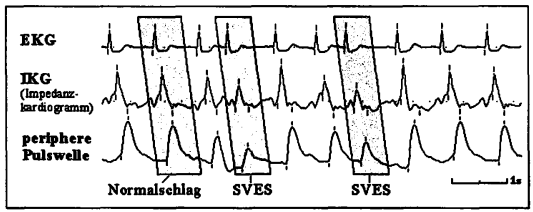

Abbildung 1: SVES mit reduzierter hämodynamischer Wirksamkeit

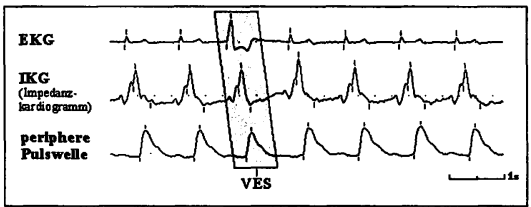

Abbildung 2: VES mit guter hämodynamischer Wirksamkeit

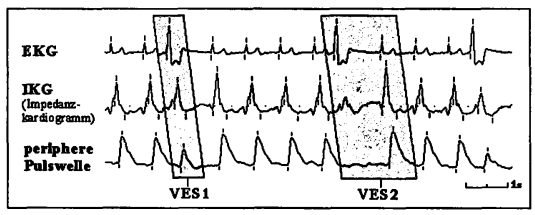

Abbildung 3: VES mit vermindertem (VES1) bzw. ohne erkennbares Schlagvolumen (VES2); deutlich erhöhtes Schlagvolumen nach VES2 
Außerdem zeigten sich bei Extrasystolen mit stark vermindertem Auswurf im thorakalen Impedanzsignal oftmals deutliche Veränderungen der gesamten Kurvenform, während im peripheren Signal meist nur eine Verringerung der Gipfelamplitude zu beobachten ist.

$\mathrm{Da}$ bei der Mehrheit der Patienten die hämodynamische Wirksamkeit arrhythmogener Schläge deutlich reduziert ist, erfolgte anhand des Schlagvolumens (SV) und der peripheren Gipfelamplitude (GA) eine quantitative Analyse der Auswurfleistung von Extrasystolen im Vergleich zu Normalschlägen. Abb. 4 zeigt anhand eines repräsentativen Beispieles die Verteilung dieser Parameter. Sowohl das mittlere Schlagvolumen als auch die mittlere Gipfelamplitude der peripheren Pulskurven waren bei VES gegenüber den Normalschlägen deutlich vermindert. Die Analyse des Impedanzkardiogramms und der peripheren Pulswelle liefert vergleichbare Ergebnisse.
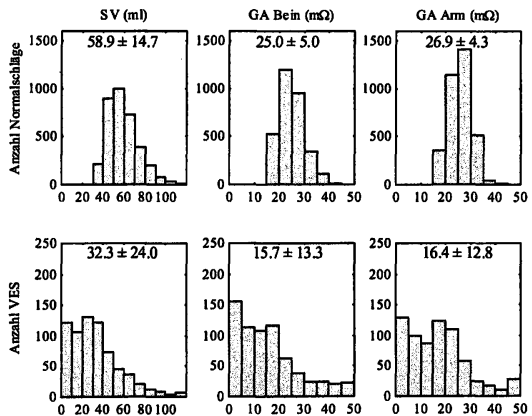

Abbildung 4: Histogramm kardialer und peripherer hämodynamischer Parameter bei Normalschlägen und VES

Auch bei ventrikulären Couplets konnte sowohl ein normaler, reduzierter als auch fehlender Auswurf beobachtet werden. Meistens hatte jedoch nur eine der beiden konsekutiven VES ein relevantes Schlagvolumen (Abb. 5).

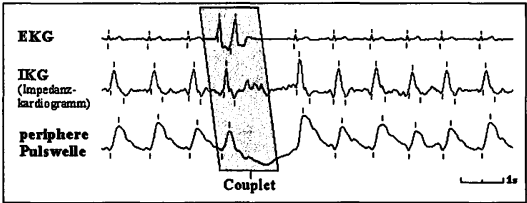

Abbildung 5: Hämodynamik bei ventrikulärem Couplet

\section{DISKUSSION}

Aufgrund der hohen intra- und interindividuellen Variabilität des von SVES und VES hervorgerufenen Schlagvolumens kann unter alleiniger Berücksichtigung des EKG keine Bewertung ihrer hämodynamischen Effektivität erfolgen. Insbesondere die bei unseren Untersuchungen festgestellte hämodynamische Ineffektivität von SVES sollte in der klinischen Routine mehr Beachtung finden.

Bei Extrasystolen mit reduziertem Auswurf kommt es im Impedanzkardiogramm aufgrund dessen komplexer Entstehung [1] oftmals zu schwer zu interpretierenden Kurvenformen. Daher sollten in diesen Fällen für eine Abgrenzung zwischen Pulskurven mit normalem oder reduziertem Volumen zusätzlich Parameter der peripheren Pulswelle, wie z.B. die Gipfelamplitude, herangezogen werden.

\section{SCHLUSSFOLGERUNGEN}

Eine klinisch relevante Einschätzung der hämodynamischen Wirksamkeit von Extrasystolen kann nur durch die kombinierte Analyse von kardialer und peripherer Pulswelle erfolgen. In Ergänzung zum LangzeitEKG liefert die Bewertung der hämodynamischen Wirksamkeit von Extrasystolen wertvolle klinische Informationen zur Beurteilung der Langzeitprognose der Patienten sowie zur Therapiebedürftigkeit der Arrhythmien.

\section{LITERATURHINWEISE}

[1] K. Churt, "Ein Beitrag zur rechnergestützten Bestimmung hämodynamischer Parameter am Thorax mittels Impedanzkardiographie", Dissertation, TU Ilmenau, 1995. 